\title{
Suivi de la qualité des effluents de la ville de Nouakchott irrigués au niveau du périmètre maraîcher de Sebkha
}

\author{
Abdoulaye DEMBA N'DIAYE ${ }^{1^{*}}$, Mohamed Ould sid'ahmed Ould KANKOU ${ }^{2}$, \\ Aminata DIASSE SARR ${ }^{3}$ et Baidy LO $^{1}$ \\ ${ }^{1}$ Laboratoire de Chimie de l'Eau, Institut National de Recherches en Santé Publique de Nouakchott, BP 690, \\ Mauritanie. \\ ${ }^{2}$ Laboratoire de Chimie de l'Eau et Environnement, Faculté des Sciences et Techniques de l'Université de \\ Nouakchott-BP 5026, Mauritanie. \\ ${ }^{3}$ Laboratoire de Chimie Inorganique, Faculté des Sciences et Techniques de l'Université Cheikh Anta Diop de \\ Dakar, Sénégal. \\ *Auteur correspondant, E-mail: abdouldemba@yahoo.fr, Tél.: 002227419103
}

\section{RESUME}

L'étude de la qualité physicochimique des effluents de la ville de Nouakchott s'est déroulée au cours du mois de mars et avril 2009. Les paramètres étudiés sont la température, le pH, la conductivité, des chlorures, matières en suspension, calcium, magnésium, sodium, potassium, bicarbonates, nitrates, ammonium, orthophosphates, sulfates, fer et chrome. Les résultats des analyses présentés dans ce travail ont révélé que ces eaux usées ont des températures pouvant atteindre $26,3{ }^{\circ} \mathrm{C}$. Le $\mathrm{pH}$ varie entre 6,91 et 7,76 . L'existence d'une forte salinité avec des valeurs maximale en conductivité électrique en chlorures et sodium respectives de 5,5 $\mathrm{mS} / \mathrm{cm}, 1633 \mathrm{mg} / \mathrm{l}$ et $390 \mathrm{mg} / \mathrm{l}$. La totalité des prélèvements ont un rapport d'adsorption de sodium inférieur à 10 donc un risque minimal d'accumulation du sodium. Les nitrates et ammonium présentent des teneurs maximales respectives de 3,50 mg/l et 292,02 mg/l confirmant l'abondance de la forme ammoniacale dans les eaux usées. La présence des ions orthphosphates, sulfates, potassium, calcium et magnésium à des teneurs variables pouvant être bénéfiques pour les cultures et la présence du fer et du chrome dont les teneurs maximales pouvant atteindre respectivement $542 \mu \mathrm{g} / \mathrm{l}$ et $368 \mu \mathrm{g} / \mathrm{l}$. L'application de l'analyse en composante principale sur ces résultats fait apparaître, globalement, deux sortes d'effluents irrigués dans le périmètre maraîcher de Sebkha: des effluents industriels présentant une forte salinité et des effluents domestiques présentant des teneurs élevées en ammonium.

(C) 2010 International Formulae Group. All rights reserved.

Mots clés: Eau usée, physicochimique, salinité, Mauritanie.

\section{INTRODUCTION}

Une étude mondiale sur la pratique cachée de l'irrigation par les eaux usées révèle que les eaux des égouts, souvent non traitées, sont utilisées pour la production de $10 \%$ des récoltes dans le monde, surtout dans les zones urbaines (Scott et al., 2004).
De nombreuses études ont été conduites au niveau du périmètre maraîcher de Sebkha (Schneider et Gagneux, 1997 ; Azandosessi et al., 1999). Elles ont mis en évidence une pollution microbiologique des eaux usées et les légumes. La présente étude se propose d'une part de suivre la qualité 
physicochimique et chimique des effluents de la ville de Nouakchott irrigués dans le périmètre maraîcher de Sebkha, d'autre part d'évaluer ces eaux usées en vue de déterminer les risques liés à l'irrigation sur la base des procédés analytiques et statistiques.

\section{MATERIEL ET METHODES Milieu d'étude}

Nouakchott est une ville côtière située vers $18^{\circ} 07$ de latitude nord et $16^{\circ} 01$ de longitude ouest (Figure 1). Le climat y est généralement sec pendant toute l'année avec des pluies faibles et très irrégulières durant l'été. Les températures oscillent entre $28,4^{\circ} \mathrm{C}$ et $36,4{ }^{\circ} \mathrm{C}$ pour les maxima et $14,6{ }^{\circ} \mathrm{C}$ et 25,7 ${ }^{\circ} \mathrm{C}$ pour les minima (Mint El Bezeid, 2007).

\section{Site d'étude}

On a choisi comme site du prélèvement la STEP (Station de Traitement des Eaux Polluées). Le réseau fonctionnel est d'une longueur de $38 \mathrm{~km}$, desservant quelques quartiers (environ 1800 ménages). La SOBOMA (Société de Boissons de Mauritanie) fabriquant des boissons gazeuses telle que Coca Cola et la Pro Pêche (une société de pêche spécialisée dans le domaine de vente de céphalopodes poulpes et seiches) sont connectées aussi à la STEP. La STEP est constituée par les étapes suivantes: 1Dégrillage : c'est une opération indispensable de rétention des matériaux encombrant tels que les substances de grosse taille, des sachets, fragments de bois. 2- Déssablage dont le but est de séparer uniquement le sable et les matériaux lourds des matières décantables les plus légères. 3- Filtration : elle consiste à absorber sur un support les particules en suspension. 4- Chloration: c'est un phénomène qui utilise le chlore dont le but est de détruire les microorganismes. 5Neutralisation: c'est une opération qui consiste à l'élimination d'alcalis forts ou acides forts dans une eau suivant l'agressivité de l'eau. 6- Décantation qui consiste au dépôt des particules en suspension présentes dans l'eau naturellement. Les bassins circulaires : les eaux usées sont distribuées au centre du bassin et se développe au-dessus du bord. La distribution s'effectue de façon uniforme et en parallèle avec l'écoulement sur les bords du bassin. Le traitement biologique utilisé est le procédé à boue activée et le procédé consiste à provoquer le développement d'une culture bactérienne dispersée sous forme de flocons (boue activée) et aliment en eau épurée. Selon les carnets d'exploitation de l'exploitant, les débits en entrée de station sont les suivants: $444 \mathrm{~m}^{3} / \mathrm{j}$ par temps sec, $458 \mathrm{~m}^{3} / \mathrm{j}$ en moyenne journalière, $1260 \mathrm{~m}^{3} / \mathrm{j}$ en débit de pointe (capacité théorique moyenne $2000 \mathrm{~m}^{3} / \mathrm{j}$ ) (AMEXTIPE, 2000). Les eaux usées brutes provenant des unités industrielles connectées à la STEP et les rejets domestiques arrivant à la STEP subissent des séries de décantation (actuellement la chloration ne se fait plus) avant d'être évacuées vers le réseau de distribution sur le site de maraîchage de Sebkha car depuis une décennie, la STEP n'est pas fonctionnelle (Cissé et Tanner, 2000).

\section{Méthodes d'analyses}

Les paramètres physicochimiques sont déterminés à partir de prélèvements hebdomadaires effectués au niveau de la sortie de la STEP au cours du mois de mars et le mois d'avril 2009. Il s'agit de trois types de prélèvements hebdomadaires. La première campagne de prélèvements a lieu les $1^{\mathrm{er}}, 8,15$, 22 et le 29 mars 2009. La deuxième campagne de prélèvement a eu lieu les 5, 12, 19 et 26 avril 2009. Les paramètres étudiés sont la température, le $\mathrm{pH}$, la conductivité, les matières en suspension, les ions chlorures, bicarbonates, calcium, magnésium, sodium, potassium, sulfates, nitrates, ammonium, orthophosphates, fer et du chrome. Le pH et la température ont été déterminés par un $\mathrm{pH}$ mètre modéle Hanna Instruments muni d'une sonde mesurant la température. La conductivité électrique a été mesurée par un conductimètre modèle Hanna Instruments. 
Les matières en suspension sont déterminées par filtration d'un volume d'eau usée sur filtre $(0,45 \mu \mathrm{m})$ selon Rodier (1996). Les chlorures sont mesurés par méthode de Mohr. Les bicarbonates sont analysés par dosage volumétrique avec du $\mathrm{HCl} 0,1 \mathrm{~N}$. Les ions calcium et magnésium sont dosés par la méthode volumétrique avec EDTA $0,02 \mathrm{~N}$. Les ions sodium et potassium sont dosés par un photomètre à émission atomique de modèle Corning. Les ions sulfates, nitrates, ammonium, orthophosphates, fer et chrome sont analysés par des méthodes colorimétriques à l'aide d'un spectrophotomètre UV Visible modéle $722 \mathrm{~S}$ Beijing. Les ions sulfates sont précipités en milieu aqueux par le baryum en présence de thymol et de gélatine. Les ions nitrates sont dosés par la méthode de salicylate. Les ions ammonium par le réactif Nessler. Pour le dosage des orthophosphates, on utilise un réactif molybdique. Le fer est dosé par l'orthophénantroline. Le chrome total est dosé dans le milieu acide en présence de diphényle carbazone.

L'étude statistique a été basée sur l'Analyse en Composante Principale (ACP). Les matrices de corrélations intermédiaires, les corrélations entre les variables et les axes et la projection des variables dans l'espace des axes ont été obtenus avec un Logiciel XLSTAT 2010.

\section{RESULTATS \\ Evolution des paramètres physico- chimiques \\ Les résultats détaillés des analyses} physico-chimiques des effluents de la ville de Nouakchott irrigués dans le périmètre maraîcher de Sebkha sont illustrés par le Tableau 1 et 2 . Les Tableaux 1 et 2 donnent les valeurs maximales, minimales, moyennes et écart types des paramètres physicochimiques étudiés entre mars et avril 2009.

\section{Application de l'analyse en composantes principales}

Le traitement des données par l'analyse en composante principale, en utilisant comme variables la température, le $\mathrm{pH}$, la conductivité électrique, matières en suspension, les ions chlorures, bicarbonates, nitrates, ammonium, sulfates, orthophosphates, fer et chrome et comme individus les 27 prélèvements effectués au cours du mois de mars et avril 2009. On donne dans le Tableau 3 les coefficients de corrélations entre les variables et les deux premiers axes F1 et F2. Ces axes montrent une bonne répartition et représentation des variables étudiés.

Tableau 1 : Résultats des paramètres physicochimiques étudiés.

\begin{tabular}{lccccc}
\hline Paramètres & Unités & Max. & Min. & Moy. & Ecart Types \\
\hline Température & ${ }^{\circ} \mathrm{C}$ & 26,3 & 23 & 24,7 & 1,06 \\
$\mathrm{pH}$ & & 7,76 & 6,91 & 7,4 & 0,32 \\
$\mathrm{CE}$ & $\mathrm{mS} / \mathrm{cm}$ & 5,5 & 2,1 & 3,6 & 1,04 \\
$\mathrm{MES}$ & $\mathrm{mg} / 1$ & 48 & 19,72 & 37,6 & 6,42 \\
$\mathrm{Cl}^{-}$ & $\mathrm{mg} / 1$ & 1633 & 397,6 & 954,4 & 333,64 \\
$\mathrm{Na}^{+}$ & $\mathrm{mg} / 1$ & 390 & 191 & 290,7 & 70,18 \\
$\mathrm{Ca}^{2+}$ & $\mathrm{mg} / 1$ & 218,04 & 67,11 & 133,23 & 63,98 \\
$\mathrm{Mg}^{2+}$ & $\mathrm{mg} / 1$ & 94,28 & 11,10 & 58,8 & 32,95 \\
$\mathrm{HCO}_{3}^{-}$ & $\mathrm{mg} / 1$ & 1537,2 & 951,6 & 1023,99 & 132,7 \\
\hline
\end{tabular}


A. DEMBA N'DIAYE et al. / Int. J. Biol. Chem. Sci. 4(4): 966-975, 2010

Tableau 2 : Résultats des paramètres physicochimiques étudiés.

\begin{tabular}{llllll}
\hline Paramètres & Unités & Max. & Min. & Moy. & Ecart Types \\
\hline $\mathrm{NO}_{3}{ }^{-}$ & $\mathrm{mg} / \mathrm{l}$ & 0,71 & 3,50 & 1,7 & 0,96 \\
$\mathrm{NH}_{4}{ }^{+}$ & $\mathrm{mg} / 1$ & 292,05 & 119,14 & 212,5 & 61,86 \\
$\mathrm{PO}_{4}{ }^{3-}$ & $\mathrm{mg} / 1$ & 39,15 & 22,78 & 28,16 & 4,35 \\
$\mathrm{SO}_{4}{ }^{2-}$ & $\mathrm{mg} / 1$ & 53,29 & 30,64 & 43,85 & 6,54 \\
$\mathrm{~K}^{+}$ & $\mathrm{mg} / 1$ & 39 & 26 & 31,83 & 4,41 \\
$\mathrm{Fer}$ & $\mu \mathrm{g} / 1$ & 542 & 72 & 197,43 & 121,57 \\
$\mathrm{Cr}$ & $\mu \mathrm{g} / 1$ & 368 & 108 & 207,21 & 84,29 \\
\hline
\end{tabular}

Tableau 3 : Corrélations entre les variables et les facteurs.

\begin{tabular}{lcc}
\hline & F1 (37,02\%) & F2 (25,92\%) \\
\hline $\mathbf{T}$ & $-0,480$ & 0,510 \\
$\mathbf{p H}$ & $-0,066$ & $-0,783$ \\
$\mathbf{C E}$ & 0,877 & $-0,361$ \\
$\mathbf{C l}$ & 0,874 & $-0,203$ \\
$\mathbf{M E S}$ & 0,627 & $-0,012$ \\
$\mathbf{H C O}^{-}$ & 0,893 & 0,109 \\
$\mathbf{S O}_{4}{ }^{-{ }^{-}}$ & 0,388 & $-0,509$ \\
$\mathbf{N O}_{3}{ }^{-}$ & 0,140 & 0,885 \\
$\mathbf{N H}_{4}{ }^{+}$ & 0,324 & 0,834 \\
$\mathbf{P O}_{4}{ }^{-}$ & 0,642 & 0,520 \\
$\mathbf{F e r}$ & $-0,478$ & $-0,209$ \\
$\mathbf{C r}$ & $-0,754$ & 0,042 \\
\hline
\end{tabular}

Tableau 4 : Matrice des corrélations inter élémentaire.

\begin{tabular}{|c|c|c|c|c|c|c|c|c|c|c|c|c|}
\hline Variables & $\mathbf{T}$ & pH & $\mathbf{C E}$ & $\mathrm{Cl}^{-}$ & MES & $\mathrm{HCO}_{3}^{-}$ & $\mathrm{SO}_{4}{ }^{2-}$ & $\mathrm{NO}_{3}^{-}$ & $\mathbf{N H}_{4}^{+}$ & $\mathrm{PO}_{4}{ }^{3-}$ & Fer & $\mathrm{Cr}$ \\
\hline $\mathbf{T}$ & 1 & & & & & & & & & & & \\
\hline pH & $-0,587$ & 1 & & & & & & & & & & \\
\hline CE & $-0,449$ & 0,095 & 1 & & & & & & & & & \\
\hline $\mathrm{Cl}^{-}$ & $-0,288$ & 0,029 & 0,955 & 1 & & & & & & & & \\
\hline MES & $-0,260$ & $-0,125$ & 0,443 & 0,406 & 1 & & & & & & & \\
\hline $\mathrm{HCO}_{3}^{-}$ & $-0,285$ & $-0,176$ & 0,845 & 0,875 & 0,272 & 1 & & & & & & \\
\hline $\mathrm{SO}_{4}{ }^{2-}$ & $-0,748$ & 0,628 & 0,398 & 0,235 & 0,071 & 0,295 & 1 & & & & & \\
\hline $\mathrm{NO}_{3}{ }^{-}$ & 0,261 & $-0,498$ & $-0,272$ & $-0,132$ & $-0,035$ & 0,216 & $-0,089$ & 1 & & & & \\
\hline $\mathrm{NH}_{4}{ }^{+}$ & 0,003 & $-0,441$ & $-0,117$ & 0,012 & 0,086 & 0,351 & $-0,057$ & 0,932 & 1 & & & \\
\hline $\mathrm{PO}_{4}{ }^{3-}$ & $-0,274$ & $-0,144$ & 0,254 & 0,331 & 0,320 & 0,627 & 0,306 & 0,739 & 0,840 & 1 & & \\
\hline Fer & 0,254 & 0,466 & $-0,403$ & $-0,385$ & $-0,400$ & $-0,415$ & 0,318 & 0,048 & $-0,236$ & $-0,155$ & 1 & \\
\hline $\mathrm{Cr}$ & 0,103 & 0,127 & $-0,672$ & $-0,700$ & $-0,785$ & $-0,516$ & $-0,189$ & $-0,050$ & $-0,069$ & $-0,303$ & 0,182 & 1 \\
\hline
\end{tabular}




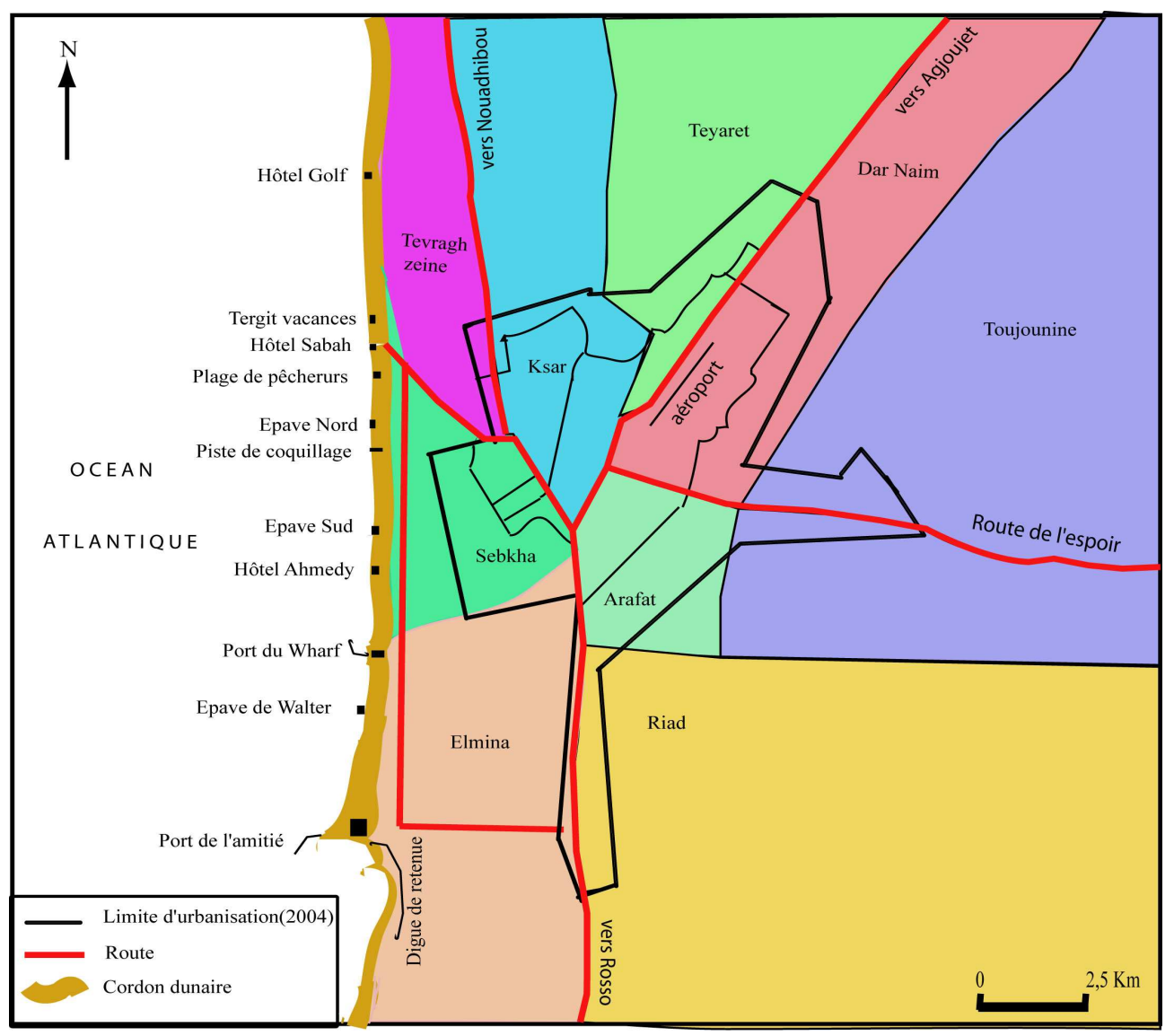

Figure 1: Présentation générale de la ville de Nouakchott.

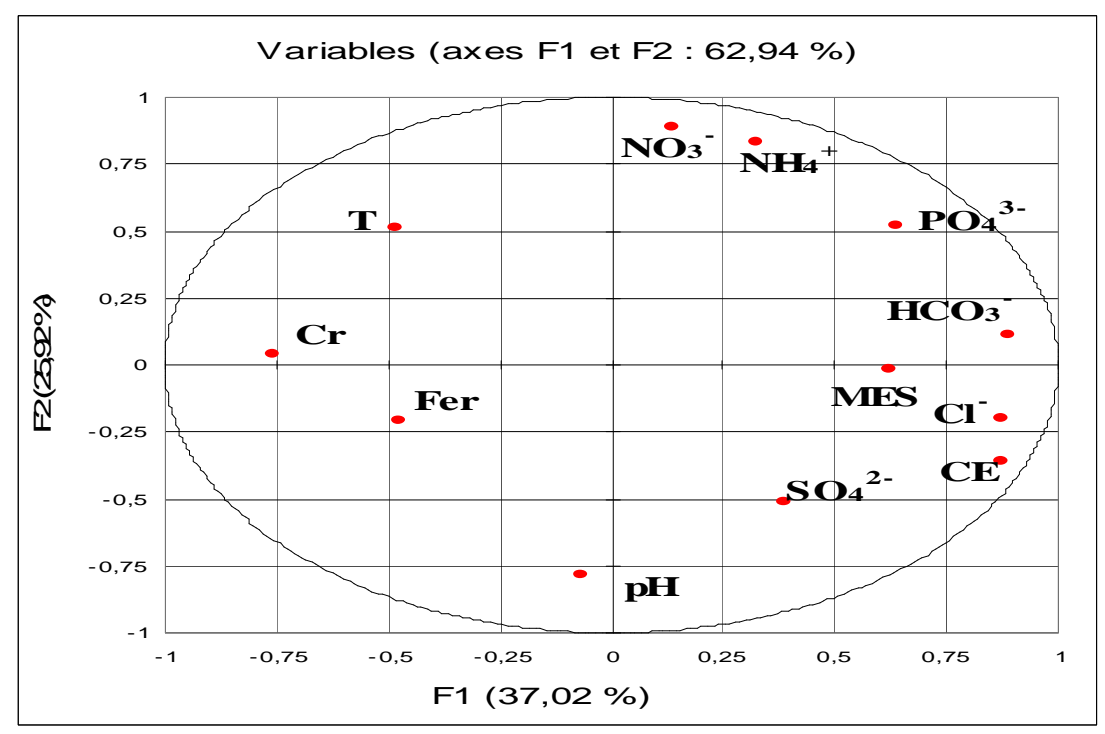

Figure 2 : Projection des variables dans l'espace des axes F1et F2. 


\section{DISCUSSION}

Il est primordial de connaître la température d'une eau. En effet, elle joue un rôle très important dans la solubilité des sels et surtout des gaz (Lynda et al., 2008). Les températures des eaux usées irriguées au niveau du périmètre maraîcher de Sebkha sont comprises entre $23{ }^{\circ} \mathrm{C}$ et $26,3{ }^{\circ} \mathrm{C}$ comme valeurs extrêmes minimales et maximales (Tableau 1). Les températures enregistrées au niveau des effluents de la STEP sont inférieures à $30{ }^{\circ} \mathrm{C}$ (Journal Officiel de la République Algérienne, 2003) et $35{ }^{\circ} \mathrm{C}$ considérée comme valeur limite de rejet direct dans le milieu récepteur (Ministère de l'Environnement du Maroc, 2002). L'analyse des prélèvements effectués au niveau de l'effluent de la SOBOMA montrent qu'il est caractérisé par une température oscillant entre $32,5{ }^{\circ} \mathrm{C}$ et $35,8{ }^{\circ} \mathrm{C}$ car la SOBOMA est équipée de plusieurs machines telle que la chaudière, la laveuse, etc. La soutireuse fonctionne en parallèle avec le rinçage des bouteilles qui passent pour le remplissage et le sertissage. Avant et après chaque séance de travail, un rinçage des machines et un nettoyage de la salle de traitement est effectué pour éliminer tous résidus liquides ou solides. Les températures des bains de rinçage des bouteilles ses situent dans un intervalle de 20 ${ }^{\circ} \mathrm{C}$ à $80{ }^{\circ} \mathrm{C}$.

Le $\mathrm{pH}$ est un élément important pour l'interprétation de la corrosion dans les canalisations des installations de l'épuration. Le $\mathrm{pH}$ des effluents de la STEP oscille entre 6,91 et 7,76. La SOBOMA utilise la soude caustique pour le nettoyage des bouteilles dont la concentration entre 0 et $3 \%$ selon les bains. Raison pour laquelle l'analyse des prélèvements effectués au niveau de l'effluent de la SOBOMA montrent que son $\mathrm{pH}$ varie entre 12,13 et 12,24 . Ce résultat est similaire à ceux trouvés par El Guamri et Belghyti (2006) au niveau des eaux usées brutes de la commune urbaine de Saknia, rejetées dans le lac Fouarat (Kenitra, Maroc).

La conductivité électrique traduit le degré de minéralisation globale. Elle nous renseigne sur le taux de salinité. C'est une expression numérique de la capacité de l'eau à conduire un courant électrique mesurée en milli siemens par centimètre. La conductivité électrique montre une large variation de la composition des effluents de la STEP, elle varie entre $2,1 \mathrm{mS} / \mathrm{cm}$ et $5,5 \mathrm{mS} / \mathrm{cm}$ (Tableau 1). Les valeurs élevées de la conductivité électrique enregistrées au niveau de la STEP pourraient être expliqués par le rejet des eaux usées résiduaires des unités industrielles connectées à la STEP. L'analyse des prélèvements effectués au niveau de la SOBOMA et de la Pro Pêche montre que leurs effluents sont caractérisés respectivement par des valeurs de la conductivité électrique oscillant respectivement de $4,4 \mathrm{mS} / \mathrm{cm}$ à 5 $\mathrm{mS} / \mathrm{cm}$ et de $29,7 \mathrm{mS} / \mathrm{cm}$ à $30,2 \mathrm{mS} / \mathrm{cm}$. Les valeurs moyennes de la conductivité trouvées au niveau de la STEP sont supérieures à celles qui sont rencontrées sur le lac de Fouarat à Kenitra au Maroc par EL Gouamri et Belghyti (2006). Par contre, les valeurs de la conductivité enregistrées sont sensiblement proches à celles trouvées à Chabat Roba, Messdour et Wadi Z'ommor en Algérie par Lynda et al. (2008). Les valeurs de la conductivité enregistrées au niveau de la STEP dépassent $2 \mathrm{mS} / \mathrm{cm}$ (Journal Officiel de la République Algérienne, 2003).

Les matières en suspension représentent l'ensemble des particules minérales et organiques contenues dans les eaux usées. Les matières en suspension représentent l'ensemble des particules minérales et organiques contenues dans les eaux usées. Leurs effets sur les caractéristiques physicochimiques de l'eau sont très néfastes (modification de la turbidité des eaux, réduction de la pénétration de la lumière dont la photosynthèse) (El Guamri et Belghyti, 2007). Les effluents de la ville de Nouakchott sont caractérisés par une concentration maximale en matière en suspension de 48 $\mathrm{mg} / \mathrm{l}$ et une valeur minimale de $19,72 \mathrm{mg} / \mathrm{l}$ (Tableau 1). La norme du rejet dans le milieu récepteur en matières en suspension est de 50 $\mathrm{mg} / \mathrm{l}$ (Ministère de l'Environnement du Maroc, 2002).

Les chlorures existent dans la quasitotalité des eaux à des concentrations très variables. Les chlorures montrent une large variation de la composition des effluents de la STEP. La valeur maximale en chlorures atteinte au niveau de la STEP est de 1633 $\mathrm{mg} / \mathrm{l}$ et la valeur minimale est de $397,6 \mathrm{mg} / \mathrm{l}$ 
(Tableau 1). Les eaux potables dans les différents quartiers de la ville de Nouakchott sont caractérisées par des teneurs en chlorures oscillant entre $106,5 \mathrm{mg} / \mathrm{l}$ et $127,8 \mathrm{mg} / \mathrm{l}$. On note une forte teneur en chlorures apportée par la Pro Pêche utilisant en grande partie de l'eau de mer pour le traitement des céphalopodes (poulpes et seiches). L'analyse des prélèvements effectués au niveau de la SOBOMA et de la Pro Pêche montre que leurs effluents sont caractérisés par des valeurs des ions chlorures oscillant respectivement de 355 $\mathrm{mg} / \mathrm{l}$ à $532,5 \mathrm{mg} / \mathrm{l}$ et de 4437,5 mg/l à 5325 $\mathrm{mg} / \mathrm{l}$. Ces concentrations trouvées au niveau des effluents de la ville de Nouakchott dépassent largement la concentration limite de rejet direct dans le milieu récepteur $(50 \mathrm{mg} / \mathrm{l})$ (Ministère de l'Environnement du Maroc, 2002).

Les effluents de la STEP sont caractérisés par des teneurs moyennes maximales et minimales respectives en bicarbonates de 1537,2 mg/l et de 951,6 mg/l (Tableau 1). Les variations de l'alcalinité des eaux usées sont rapprochées de celles du degré de minéralisation (conductivité électrique, dureté totale, $\mathrm{pH}$ ) qui donne aussi une indication sur le degré d'oxydation des composés organiques (cas des eaux résiduaires) (Thomas, 1995). Les normes marocaines recommandent une concentration en $\mathrm{HCO}_{3}^{-}$de $250 \mathrm{mg} / \mathrm{l}$ pour les eaux destinées à l'irrigation des cultures (Ministère de l'Environnement du Maroc, 2002).

Les teneurs en sodium oscillent entre $191 \mathrm{mg} / \mathrm{l}$ et $390 \mathrm{mg} / \mathrm{l}$ (Tableau 1). L'eau potable de la ville de Nouakchott est caractérisée par des teneurs en sodium oscillant entre $59 \mathrm{mg} / \mathrm{l}$ et $62 \mathrm{mg} / \mathrm{l}$. Le sodium est l'un des éléments les plus indésirables dans l'eau d'irrigation. Le problème principal avec une grande quantité de sodium est son effet sur la perméabilité du sol et sur l'infiltration de l'eau. Le sodium remplace le calcium et le magnésium adsorbés sur les particules d'argile et provoque la dispersion des particules du sol. Le sodium contribue aussi directement à la salinité totale de l'eau. Les effluents de la ville de Nouakchott présentent des teneurs moyennes en calcium et en magnésium variant respectivement de $67,11 \mathrm{mg} / \mathrm{l}$ à 226,05 mg/l (Tableau 1) et 11,10 $\mathrm{mg} / \mathrm{l}$ à 94,28 mg/l (Tableau 1). Les eaux potables dans les différents quartiers de la ville de Nouakchott sont caractérisées par des teneurs en calcium et en magnésium oscillant respectivement de $35,2 \mathrm{mg} / \mathrm{l}$ à $41,8 \mathrm{mg} / \mathrm{l}$ et $10,21 \mathrm{mg} / \mathrm{l}$ à $16,04 \mathrm{mg} / \mathrm{l}$. Les risques de sodisation relatifs à une eau d'irrigation sont caractérisés par deux paramètres : le Sodium Adsorption Ratio (SAR) qui rend compte du rapport entre les concentrations en sodium et en alcalino-terreux et la conductivité de l'effluent irrigué au niveau du périmètre maraîcher de Sebkha. Le SAR utilisé par Jiménez et Chavez (1954) est défini par la relation suivante :

$$
\mathrm{SAR}=\left[\mathrm{Na}^{2+}\right] /\left[\sqrt{ }\left(\mathrm{Ca}^{2+}+\mathrm{Mg}^{2+}\right) / 2\right]
$$

Les concentrations des ions $\mathrm{Na}^{+}, \mathrm{Ca}^{2+}$, $\mathrm{Mg}^{2+}$, sont exprimées en méq/L. Les valeurs du SAR ont été comparées à celles du diagramme de l'US Salinity Laboratory (1954), lequel, à partir des valeurs du SAR et de la conductivité, classe les eaux d'irrigation en fonction des risques de sodisation et de la sédimentation (Faby et Brissaud, 1997). Le SAR enregistré au niveau des eaux irriguées dans le périmètre maraîcher de Sebkha varie entre 5,6 et 6,3 . Tous les prélèvements ont un rapport d'adsorption de sodium inférieur à 10 , donc un risque minimal d'accumulation du sodium (Tableau 1). Le report des résultats d'analyse à l'échelle établie par l'US Salinity Laboratory (1954) montre que les eaux usées irriguées au niveau du périmètre maraîcher de Sebkha sont dans la classe $\mathrm{C}_{4} \mathrm{~S}_{1}$ c'est-à-dire que les eaux usées irriguées appartiennent à la classe $\mathrm{C}_{4}$ des conductivités, qui est la classe des eaux à très forte salinité et à la classe $S_{1}$ des eaux excellentes à faibles dangers d'alcalinisation.

Ainsi, Richards (1954) cité dans Faby et Brissaud (1997) a établi une correspondance entre la conductivité des eaux d'irrigation et la quantité de chlorures de sodium. Ainsi, une conductivité de l'effluent située entre 2250 $\mu \mathrm{S} / \mathrm{cm}$ et $5000 \mu \mathrm{S} / \mathrm{cm}$ correspondrait à une concentration en $\mathrm{NaCl}$ située entre 1500 et $3600 \mathrm{mg} / \mathrm{l}$. Une telle teneur en sel serait élevée pour l'irrigation des espaces verts. Les eaux usées irriguées dans le périmètre maraîcher de Sebkha présentent des valeurs de conductivité oscillant entre $2100 \mu \mathrm{m} / \mathrm{cm}$ et 
$5300 \mu \mathrm{S} / \mathrm{cm}$ correspondant à une concentration en $\mathrm{NaCl}$ située entre 655,2 et $2691 \mathrm{mg} / \mathrm{l}$.

Les teneurs en nitrates enregistrées au niveau de la STEP varient entre $0,71 \mathrm{mg} / \mathrm{l}$ et 3,50 $\mathrm{mg} / \mathrm{l}$ (Tableau 2). La comparaison des concentrations enregistrées au niveau de la STEP avec la norme de qualité des eaux destinées à l'irrigation montre que, ces concentrations sont très inférieures à $50 \mathrm{mg} / \mathrm{l}$, ce qui permet de déduire que ces effluents sont acceptables pour l'irrigation des cultures (Ministère de l'Environnement du Maroc, 2002). La présence des ions ammonium est à rapprocher de celle des autres éléments azotés identifiés dans l'eau (nitrate, nitrite) et des résultats de l'analyse bactériologique (Rodier, 1996). L'azote ammoniacal peut aussi provenir de ruissellements urbains, de l'agriculture (engrais) ou de l'industrie (pharmaceutique, alimentaire, pâte à papier, textile) L'urine est la principale source d'ammonium dans les eaux usées domestiques (Bonté et al., 2008). La valeur moyenne maximale en ammonium est de $292,05 \mathrm{mg} / \mathrm{l}$ et la valeur moyenne minimale est de 119,14 mg/l (Tableau 2). La grande partie du phosphore organique provient également des déchets du métabolisme des protéines et de son élimination sous forme de phosphates dans les urines par l'homme. Le phosphore n'est pas toxique intrinsèquement pour la faune et la flore terrestres et aquatiques. Par contre, l'« eutrophisation», conséquence directe majeure d'un excès de phosphore dans le milieu, a des effets très préoccupants à de nombreux niveaux (Du Chaufour, 1997). Les teneurs en orthophosphates oscillant entre $22,78 \mathrm{mg} / \mathrm{l}$ et $39,15 \mathrm{mg} / \mathrm{l}$ (Tableau 2). La concentration de 10 $\mathrm{mg} / \mathrm{l}$ en orthophosphates est une valeur limite acceptable d'un rejet direct dans le milieu récepteur (Ministère de l'Environnement du Maroc, 2002). Les teneurs en sulfates oscillant entre $53,29 \mathrm{mg} / \mathrm{l}$ et $30,64 \mathrm{mg} / \mathrm{l}$ (Tableau 2). Les teneurs en potassium $26 \mathrm{mg} / \mathrm{l}$ et $39 \mathrm{mg} / \mathrm{l}$ (Tableau 2). La présence du potassium, dans les eaux usées peut avoir un impact négatif sur la santé humaine et dans une moindre mesure un impact bénéfique sur les cultures dans le cas d'une réutilisation agricole.

Les métaux lourds que l'on trouve dans les eaux usées urbaines ont des origines multiples : ils proviennent des produits consommés au sens large par la population, de la corrosion des matériaux des réseaux de distribution d'eau et d'assainissement, des activités de service (santé, automobile) et éventuellement de rejets industriels (Cauchy et al, 1996). Les eaux usées peuvent être chargées en fer du fait des phénomènes de corrosion dans les conduites ou du matériel de fabrication (Hem, 1972 ; Olivier et al. 1975). La SOBOMA est caractérisée pardes concentrations supérieures à $0,1 \mathrm{mg} / \mathrm{L}$ de fer au niveau de l'embouteillage (prélevé dans l'égout rassemblant l'eau de rinçage des machines), la laveuse et du collecteur. Les eaux au niveau de l'embouteillage et de la laveuse montrent des concentrations élevées en fer probablement dues aux conduites en fer des eaux usées et au moment du rinçage aussi. Dans les industries alimentaires (sucrerie, brasserie, boissons gazeuses, etc.), il est recommandé de ne pas dépasser $200 \mu \mathrm{g} / \mathrm{l}$ (Rodier, 1996). La présence du chrome est le plus souvent liée aux rejets industriels provenant des ateliers de galvanoplastie, de tannage, etc. (Sepher et al., 2005). Les concentrations en fer et en chrome enregistrées au niveau des effluents de la STEP sont très variables. Les teneurs en fer et en chrome varient respectivement de $72 \mu \mathrm{g} / \mathrm{l}$ à $542 \mu \mathrm{g} / \mathrm{l}$ (Tableau 2) et de $108 \mu \mathrm{g} / \mathrm{l}$ à $368 \mu \mathrm{g} / \mathrm{l}$ (Tableau 2).

L'ACP est un outil d'analyse de données qui permet d'expliquer la structure des corrélations ou des covariances en utilisant des combinaisons linéaires des données originelles. Son utilisation permet de réduire et d'interpréter les données sur un espace réduit (Maliki, 2000). L'ACP a pour objectif de présenter, sous une forme graphique le maximum de l'information contenue dans une table de données, basées sur le principe de double projection sur les axes factoriels (Lagarde, 1995). L'analyse du plan factoriel F1 et F2 montre que plus de $62,94 \%$ sont exprimés. L'analyse dans ce plan est donc acceptable (Athamena, 2006). L'axe F1 possède une variance de $37,02 \%$ et l'axe F2 a une variance de 25,92\%. L'axe F1est exprimé par son pôle positif par la conductivité électrique, les ions chlorures, matières en suspension, bicarbonates, sulfates et orthophosphates qui présentent des 
corrélations entre eux et son pôle négatif par le fer et le chrome total (Tableau 4). L'axe F2 est constitué par la température, les ions nitrates et ammonium dans le sens positif et le $\mathrm{pH}$ dans le sens négatif. La représentation graphique des individus (échantillon ou unités statistiques) fait apparaître, globalement, deux groupes d'effluents (Figure 2). Un effluent bicarbonaté, sulfaté, phosphaté, présentant une forte salinité avec une composition métallique variable d'une part et un effluent azoté dont la forme ammoniacale est la plus abondante avec une température élevée d'autre part. Les rejets industriels sont constitués par une forte salinité due à l'utilisation de l'eau de mer pour le traitement des céphalopodes en ce qui concerne la Pro Pêche et une forte alcalinité due à l'utilisation de la soude caustique pour le rinçage des bouteilles dans le cas de la SOBOMA. Ces rejets sont caractéristiques de l'axe F1. Les rejets domestiques proviennent des différents usages domestiques de l'eau et se répartissent en eaux ménagères, qui ont pour origine les salles de bains et les cuisines et sont généralement chargées de détergents, les rejets des toilettes, chargés de diverses matières organiques azotées et de germes fécaux. Ces rejets sont caractéristiques de l'axe F2.

\section{Conclusion}

Le suivi de la qualité physicochimique et chimique des effluents de la ville de Nouakchott a montré l'existence d'une pollution se traduisant par une forte salinité pouvant avoir un impact négatif sur les sols et les cultures. Les effluents de la ville de Nouakchott appartiennent à la classe $\mathrm{C} 4$ des conductivités, qui est la classe des eaux à très fortes salinité et à la classe $S_{1}$ des eaux excellentes à faibles dangers d'alcalinisation.

L'application de l'analyse en composante principale sur ces résultats fait apparaître, globalement, deux sortes d'effluents irrigués dans le périmétre maraîcher de Sebkha: des effluents industriels présentant une forte salinité et des effluents domestiques présentant des teneurs élevées en ammonium.

La qualité physicochimique de ces eaux usées peut avoir un impact négatif sur les sols et les cultures. Il apparaît clairement que le principe d'une interdiction d'une réutilisation des eaux usées n'est pas la solution adéquate. Dès alors, il s'impose d'assujettir l'utilisation des eaux usées à des mesures d'accompagnement en vue du respect des normes de qualité des produits. Ces mesures tournent autour de l'épuration des eaux usées avant leur réutilisation.

\section{REFERENCES}

AMEXTIPE. 2000. Etude sur la Stratégie de Développement des villes de Nouakchott, Nouadhibou et Kaédi (Volet: Evaluation Environnementale et Sociale Stratégique), Rapport préliminaire A 2003, République Islamique de Mauritanie.

Athamena M. 2006. Etude des ressources thermales de l'ensemble allochtone su Sétifien. Thèse de Magister, Université de Batna, p. 131.

Azandossessi A, Ould Selmane ML, Ould Baba L, Benzeroug EH, Cissé G et Tanner M. 1999. Projet de préservation de l'unique espace vert de Nouakchott : le site de Sebkha « Nouakchott El Khadra ». Document de projet. OMS, Nouakchott.

Bonté SL, Pons M, Potier O, Rocklin P. 2008. Relation between conductivity and ion content in urban wastewater. Journal of Water Science, 21(4): 429- 438.

Cauchi, Hyvrard, Nakache, Schwarzbrod, Zagury, Baron, Carré, Courtois, Denis, Dernat, Larbaigt, Dérangére, Martigne, Séguret. 1996. Dossier : la réutilisation des eaux usées après épuration. Techniques, Sciences et Méthodes, 2: 81118

Cissé G, Tanner M. 2000. Analyse de la situation de l'agriculture à Nouakchott (Mauritanie) et à Ouagadougou (Burkina Faso), Conférence électronique RUAF.

DU Chaufour P. 1997. Abrégé de Pédologie: Sol, Végétation et Environnement (5è édn). Masson.

EL Guamri YD, Belghyti D. 2006. "Etude de la qualité physico-chimique des eaux usées brutes de la commune urbaine de Saknia, rejetées dans le lac Fouarat (Kénitra, Maroc)". Journal Africain des Sciences de l'Environnement, 1: 53-60.

EL Guamri YD, Belghyti D. 2007. "Etude de l'aspect physico-chimique et parasitolo- 
gique des eaux usées destinées à l'irrigation de périmètre péri-urbain de Fouarat (Kénitra, Maroc)". Revue Agronomie Africaine, 19(3): 251 - 261.

Faby JA, Brissaud F. 1997. L'utilisation des eaux usées épurées en irrigation. Compte rendu d'étude de l'Office International de l'Eau, mai 1997, 82 p.

Hem JD. 1972. Chemical factors that influence the availability of iron and manganese in aqueous systems. Geol. Soc. Am. Spec. Pap., 140: 17-24.

Jimenez CB, Chavez MA.1997. Treatment of Mexico for irrigation purposes. Environ. Technol., 18 : 721-729.

Journal Officiel de la République Algérienne. 1993. Normes de rejets dans le milieu récepteur. Journal Officiel de la République Algérienne, 46 : 7-12.

Lagarde J. 1995. Initiation à l'Analyse des Données. Ed. Dunod : Paris; 157 p.

Lynda S, Rachid R, Houria B and Mohammed-Reda D. 2008. African Journal of Environnemental Science and Technology, 2(8): 231-238.

Maliki AM. 2000. Etude hydrologique hydro chimique et isotopique de la nappe profonde de Sfax. Thèse de Doctorat, Sfax, Tunisie, $301 \mathrm{p}$.

Ministère de l'Environnement du Maroc. 2002. « Normes marocaines, Bulletin officiel du Maroc », $\mathrm{N}^{\circ} 5062$ du 30 ramadan 1423. Rabat.

Mint El Bezeid F. 2007. Evaluation de risques Environnement qui menacent la zone côtière de Nouakchott et les solutions possibles (Mauritanie) Mémoire DESA, Université Chouaib Doukkali, Maroc.
Oliver BG, Cosgroove EG.1975. Metal concentrations in the sewage, effluents and sludges of some southern Ontario wastewater treatment plants, Environ. Lett., 9: 75 .

Richards LA. 1954. Diagnosis and Improvement of Saline and Alkali Soils. USDA Agricultural Handbook, $\mathrm{N}^{\circ} 60$, US Department of Agriculture: Washington, DC, Etats- Unis; 160 p.

Rodier J. 1996. L'Analyse de l'Eau Naturelle, Eaux Résiduaires, Eau de Mer (8 éme édn). Denod: Paris; 1383 p.

Schneider C, Gagneux S. 1997. Impact sanitaire de l'utilisation d'eaux usées et polluées en agriculture urbaine: Cas du maraîchage à Nouakchott, République. Islamique de Mauritanie. Travail de Diplôme, Institut Tropical Suisse, Université de Bâle.

Scott CA, Farauqui NI, Sally RL. 2004. Wastewater use in irrigated agriculture management challenge in developing countries, IWMI, CRDI, 9 p.

Sepher MN, Nasseri S, Assadi MM, Yaghmalan K. 2005. Chromium bioremoval from tannery industries effluent by Aspergillus Oryzae. Iran. J. Environ. Health. Sci. Eng., 2: 273-279.

Thomas O. 1995. Métrologie des Eaux Résiduaire. Ed. Cebedoc / Tec. et Doc. 11 : Liège -75384, Paris.

US Salinity Laboratory. 1954. Diagnosis and Improvement of Saline and Alkali Soils. USDA Agriculture Handbook 60. Washington, DC, Etats-Unis, US Government Printing Office; 10-50. 